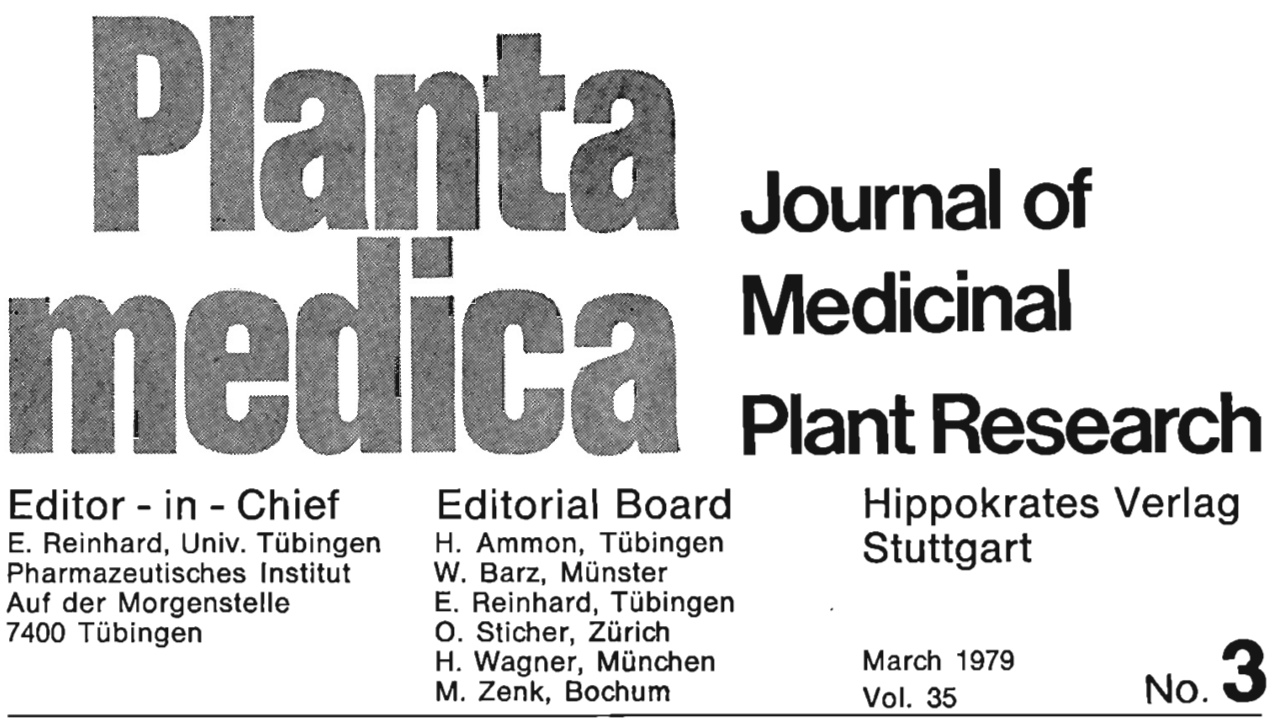

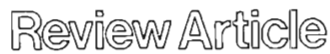

\title{
Neue Enzyminhibitoren aus Mikroorganismen
}

\section{New Enzyme Inhibitors from Microorganisms}

W. Frommer, B. Junge, L. Müller, D. Schmidt und E. Truscheit

Bayer AG, Pharma Forschungszentrum, 5600 Wuppertal 1, Bundesrepublik Deutschland.

Key Word Index: Enzyme Inhibitors; Protease Inhibitors; Amylase Inhibitors; Sucrase Inhibitors; Microorganisms; Actinomycetes.

\section{Abstract}

A literature survey on the structures and specificities of enzyme inhibitors isolated from microorganisms is presented, with special emphasis on protease inhibitors and inhibitors of adrenaline metabolism.
In addition a new group of glycoside hydrolase inhibitors isolated from actinomycetes is discussed in detail, with special reference to detection, production, pharmacological effects and structure of the inhibitors. An integral part in the chain of $\alpha-1,4$-linked D-glucopyranose units is a core structure consisting 
of an unsaturated cyclitol unit bound to 4,6-dideoxy-4-amino-D-glucopyranose.

The low molecular weight homologues are potent sucrase inhibitors whereas the inhibitors of higher molecular weight are specific for $\alpha$-amylase.

\section{Einleitung}

Die klassischen Wirkstoffe aus Mikroorganismen sind die Antibiotika. Bis heute sind ca. 4500 verschiedene Antibiotika beschrieben worden, und Jahr für Jahr kommen z. Z. 250-300 neue dazu [1]. Der Aufwand, neue Antibiotika zu finden, wird vor allem durch die Differenzierung zu den bereits bekannten Antibiotika immer größer. Trotzdem hat man, wie die Arbeiten der letzten Jahre zeigen, auch heute immer noch die Chance, neue interessante Verbindungsklassen $\mathrm{zu}$ isolieren, vor allem, wenn man bei der Suche neue Wege geht.

Antibiotika kann man meist durch einfache Bakterienhemmteste, so wie sie anfallen, z. B. in Kulturbrühen nachweisen. Diese leichte Nachweisbarkeit ist sicher der Grund dafür, daß wir heute soviel über Antibiotika wissen.

Die Antibiotika greifen an sehr vielen verschiedenen, aber essentiellen Stellen im Stoffwechsel ein. Man kann die Frage stellen, ob Mikroorganismen auch andere Wirkstoffe bilden, die an periphereren, nicht essentiellen Stoff wechselbahnen eingreifen. Diese Stoffe kann man nicht mit Antibiotikahemmtesten erfassen. Man muß andere Suchteste anwenden, um sie zu finden.

UMEZA WA [2] hat in der 2. Hälfte der 60er Jahre angefangen, spezifische Inhibitoren vor allem von Proteasen und Inhibitoren von Enzymen der AdrenalinBiosynthese zu suchen. Parallel dazu haben wir versucht, therapeutisch anwendbare Inhibitoren von Glycosid-Hydrolasen zu finden [3].

Diese Zusammenfassung soll im ersten Teil eine Ưbersicht über das Vorkommen und die Art dieser Enzym-Inhibitoren aus Mikroorganismen geben und soll im 2. Teil den Stand unserer Arbeiten über Inhibitoren von Glycosid-Hydrolasen, vorwiegend von solchen des Verdauungstraktes von Säugetieren, darstellen.

\section{Proteasen-Inhibitoren}

Proteasen-Inhibitoren sind in Tieren und Pflanzen weit verbreitet [4]. Sie übernehmen in vielen Fällen, z. B. bei der Blutgerinnung, eine wichtige Regulationsfunktion. Alle diese Stoffe stellen Oligopeptide oder Polypeptide, z. T. auch Glycoproteide dar. Im Verlauf der 60er Jahre erschienen die ersten Arbeiten, die einzelne Proteasen-inhibitorische Wirkungen aus Mikroorganismen beschrieben, so aus Clostridium botulinum [5], aus E. coli [6], aus unbestimmten Gram-negativen Bakterien [7] und aus Penicillien und Aspergillen [8].

UMEZAwa u. Mitarbeiter gingen bei ihren systematischen Arbeiten, Proteasen-Inhibitoren in Mikroorganismen zu suchen, davon aus, daß es für Mikroorganismen nützlich sein sollte, ihre Proteasen dann zu hemmen, wenn sie nicht oder nicht mehr gebraucht werden. Sie fanden gewissermaßen im ersten Anlauf in Kulturen vieler Arten von Strep- 


\section{Tabelle I}

Inhibitoren von proteolytischen Enzymen und ihre Herkunft (H. Umezawa)

Inhibitoren Streptomyces species

Leupeptine

S. roseus, S. roseochromogenes

S. lavendulae, S. albireticuli,

S. thioluteus, S. chartreusis, und weitere 11 Spezies

Chymostatine S. hygroscopicus, S. lavendulae etc.

Pepstatine S. testaceus, S. argenteolus etc.

Antipain S. mauvecolor, A. violascens, S. michiganensis

S. yokosukanensis

tomyceten die Leupeptine [9], das Anti- Peptide.

pain [10], die Chymostatine [11] und die Pepstatine $[12,13]$.

Die Leupeptine bestehen aus 2 Haupt-

Die Tab. I zeigt die weite Verbrei- pionyl-L-leucyl-L-leucyl-D, L-arginal tung dieser Stoffe nach einer Zusammenstellung aus dem Jahre 1974 [14].

Bei allen 4 Stoffen bzw. Stoffgruppen handelt es sich um niedermolekulare [13] (Abb. 1).

Das Antipain ist ein (S)-1-Carboxy2-phenyläthyl-carbamoyl-L-arginyl-Lvalyl-arginal nach [13] (Abb. 2).

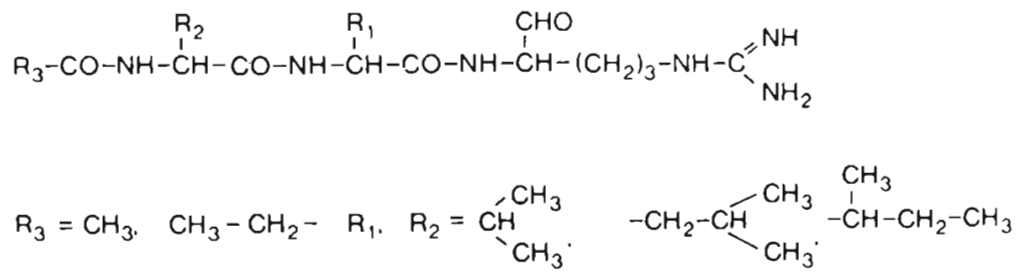

Leupeptin AC-LL: $\mathrm{R}_{3}=\mathrm{CH}_{3}-, \mathrm{R}_{1}, \mathrm{R}_{2}=-\mathrm{CH}_{2}-\mathrm{CH} \underset{\mathrm{CH}_{3}}{\mathrm{CH}_{3}}$

Leupeptin Pr-LL: $\mathrm{R}_{3}=\mathrm{CH}_{3}-\mathrm{CH}_{2}-. \mathrm{R}_{1} \cdot \mathrm{R}_{2}=-\mathrm{CH}_{2}-\mathrm{CH}_{\mathrm{CH}_{3}}^{-\mathrm{CH}_{3}}$

Abb. 1. Struktur der Leupeptine (H. UmEZA A)

Abb. 2. Antipain

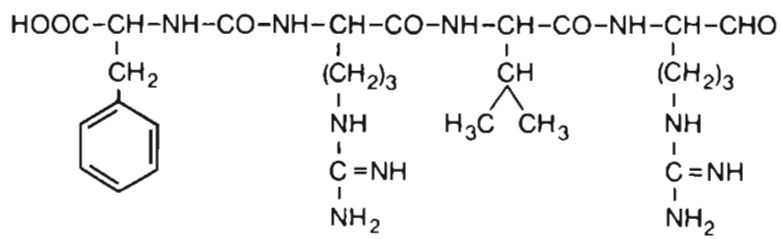


Die Fähigkeit zur Bildung von Antipain ist unter den Actinomyceten sehr weit verbreitet. Wir selbst fanden Antipain auch bei Planomonospora parontospora [15].

Chymostatin [16] ist ebenfalls ein Gemisch aus Peptiden mit Phenylalaninal an einem Ende (Abb. 3).

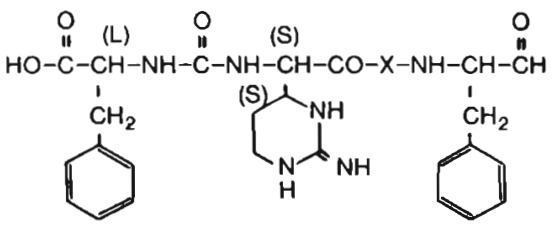

Chymostatin A:X $=$ L-Leu

Chymostatin B. $X=L-$ Val

Chymostatin $\mathrm{C}: \mathrm{X}=\mathrm{L}-\| \mathrm{le}$

Abb. 3. Chymostatine
Die Struktur der Pepstatine [13] zeigt Abb. 4. Es handelt sich um Pentapeptide aus L-Alanin, zwei Molen L-Valin und zwei Molen einer neuen Aminosäure 4Amino-3-hydroxy-6-methylheptansäure.

Die sehr spezifische Wirkung dieser Inhibitoren zeigt die Tab. II [14]. Die Leupetine hemmen vorwiegend Trypsin, Papain, Plasmin und Cathepsin B, das Antipain Papain, Trypsin und die Cathepsine $\mathrm{A}+\mathrm{B}$, das Chymostatin vorwiegend Chymotrypsin und Papain und die Pepstatine Pepsin und Cathep$\sin \mathrm{D}$.

$\mathrm{Zu}$ diesen heute schon als nahezu klassisch geltenden Proteasen-Inhibitoren kam in den letzten Jahren eine ganze Reihe weiterer hinzu.

\section{Tabelle II}

Wirkung von Leupeptin, Pepstatin, Chymostatin und Antipain gegen verschiedene Proteasen

\begin{tabular}{llcccc}
\hline Enzyme & Substrate & Leupeptin & $\begin{array}{l}\mathrm{ID}_{50}(\mu \mathrm{g} / \mathrm{ml}) \\
\text { Pepstatin }\end{array}$ & Chymostatin & Antipain \\
\hline Thrombokinase & Plasma & 15 & $>250$ & $>250$ & 20 \\
Thrombin & TAME $^{1}$ & 10,000 & $>250$ & $>250$ & $>250$ \\
Plasmin & Fibrinogen & 8 & $>250$ & $>250$ & 93 \\
Trypsin & Casein & 2 & $>250$ & $>250$ & 0,26 \\
Papain & Casein & 0,5 & $>250$ & 7,5 & 0,16 \\
Kallikrein & BAEE & 75 & $>250$ & $>250$ & $>250$ \\
$\alpha$-Chymotrypsin & Casein & $>500$ & $>250$ & 0,15 & $>250$ \\
$\beta, \gamma$ and $\delta$-Chymotrypsin & Casein & $>500$ & $>250$ & 0,15 & $>250$ \\
Pepsin & Casein & $>500$ & 0,01 & $>250$ & $>250$ \\
& Hemoglobin & $>500$ & 0,0031 & $>250$ & $>250$ \\
Proctase A & Casein & $>250$ & $>250$ & $>250$ & $>250$ \\
Proctase B & Casein & $>250$ & 0,0072 & 26,5 & 190 \\
Cathepsin A & Cb-Glut-Tyr & 1,680 & $>125$ & 62,5 & \\
Cathepsin B & BAA & 0,44 & $>125$ & 2,6 & \\
Cathepsin D & Hemoglobin & 109 & 0,011 & 49,0 & \\
& & & &
\end{tabular}

1 a-N-(p-Toluolsulfonyl)- $\mathrm{L}$-argininmethylester $\mathrm{HCl}$

2 a-N-Benzoyl-L-argininethylester $\mathrm{HCl}$

3 a-N-Carbobenzoxy-L-glutamyl-L-tyrosin

4 a-N-Benzoyl-L-argininamid $\mathrm{HCl}$ 
Ein Teil dieser in den letzten Jahren beschriebenen Stoffe zeigt Ahnlichkeiten mit den bereits bekannten, so ein dem Antipain-ähnlicher Inhibitor KF 77-AG 6 [17] und eine Reihe zur PepstatinGruppe gehörender Stoffe, die alle 4Amino-3-hydroxy-6-methylheptansäure enthalten, wie die Pepstatine $B U, P R$, AC mit $R_{1}=$ Butyryl-, Propionyl-, Acetyl-[18], das Hydroxypepstatin mit Serin anstelle von Alanin [19], die Pepsinostreptine mit $\mathrm{R}_{1}=$ Propionyl- oder Isobutyryl-, als freie Säure und als Methylester [20] und die Procidine, bei denen einer oder beide Valin-Reste durch Leucin ersetzt sind [21].

Ein anderer Teil zeigt vor allem vollständig neue Spezifitäten. Plasminostreptin, ein Polypeptid aus 109 Aminosäuren, stammt von Streptomyces anti- fibrinolyticus und zeigt eine spezifische Wirkung gegen Plasmin, Trypsin und alkalische Proteasen [22].

Weitere Inhibitoren von alkalischen Proteasen aus Mikroorganismen beschreiben Murao et al. [23]. Die Struktur eines dieser Stoffe, des Subtilis-Inhibitors, ist vollständig beschrieben. Es handelt sich um eine Polypeptid mit 113 Aminosäuren, das in der Aminosäuresequenz Ahnlichkeit mit dem sekretorischen Trypsin-Inhibitor (KazalInhibitor) aus Rinderpankreas und dem Plasminostreptin zeigt [24].

Auch aus Hefen wurden verschiedene Proteasen-Inhibitoren mit Polypeptidcharakter (MG 9-10000, 23000 und 27000) isoliert, die einzelne in der Hefezelle vorkommende Proteasen A, B und C selektiv hemmen [25].<smiles>[R3]CC([R2])C(CC(C)C)NC(=O)C(C)NC(=O)CC(O)C(CC(C)C)NOC(=O)C(NC(=O)C(N[R7])C(C)C)C(C)C</smiles>

\begin{tabular}{|c|c|c|c|}
\hline & $R_{1}$ & $\mathrm{R}_{2}$ & $R_{3}$ \\
\hline Pepstatin A & iso-valeryl & $=\frac{\mathrm{H}}{\mathrm{OH}}$ & $-\mathrm{COOH}$ \\
\hline Pepstatin B & n-caproyl & $=\frac{\mathrm{H}}{\mathrm{OH}}$ & $-\mathrm{COOH}$ \\
\hline Pepstatin C & iso-caproyl & $\mathrm{S}_{\mathrm{OH}}^{\mathrm{H}}$ & $-\mathrm{COOH}$ \\
\hline Pepstatin D & n-heptanoyl & $\begin{array}{l}\mathrm{H} \\
\mathrm{OH}\end{array}$ & $-\mathrm{COOH}$ \\
\hline Pepstatin E & iso-heptanoyl & ${ }_{\mathrm{OH}}^{\mathrm{H}}$ & $-\mathrm{COOH}$ \\
\hline Pepstatin F & anteiso-heptanoyl & $=\stackrel{\mathrm{H}}{\mathrm{OH}}$ & $-\mathrm{COOH}$ \\
\hline Pepstatin G & n-capryloyl & $\mathrm{S}_{\mathrm{OH}}^{\mathrm{H}}$ & $-\mathrm{COOH}$ \\
\hline Pepstatin H & iso-capryloyl & $\mathrm{S}_{\mathrm{OH}}^{\mathrm{H}}$ & $-\mathrm{COOH}$ \\
\hline epstanone A & iso-valeryl & $=0$ & $-\mathrm{H}$ \\
\hline
\end{tabular}

$A b b$. 4. Struktur der Pepstatine 


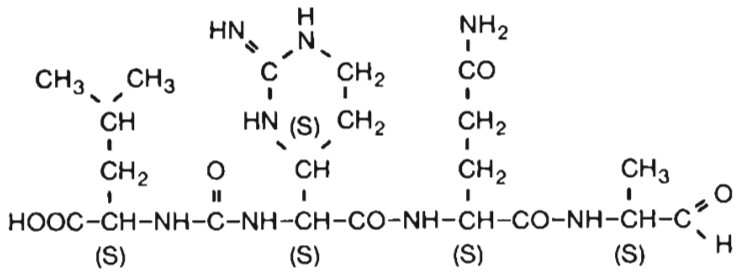

$A b b$. 5. Struktur des Elastatinal. N-[(S)-1-Carboxy-isopentyl] carbamoyl- $\alpha$-[2-iminohexahydro-4 (S)-pyrimidyl]-(S)-glycyl-(S)-glutaminol-(S)-alaninal

Das Elastatinal [26] hemmt spezifisch die Pankreas-Elastase. Die Strukturformel gibt die Abb. 5 [27] wieder. Das Elasnin [76] hemmt bevorzugt die Granulocyten-Elastase.

Das Phosphoramidon ist ein spezifischer Hemmstoff des Thermolysins, einer Metalloendopeptidase aus Bacillus thermoproteolyticus [28]. Die Struktur zeigt die Abb. 6.

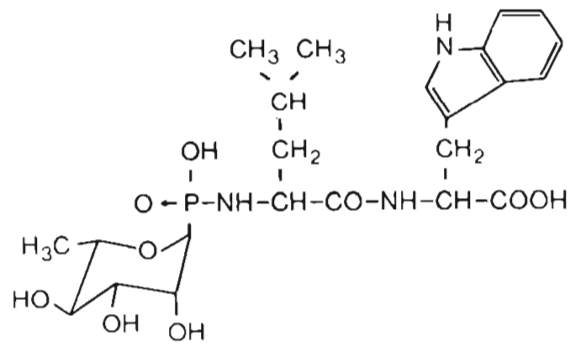

Abb. 6. Phosphoramidon

Einen spezifischen Inhibitor für Aminopeptidase B aus Rattenleber stellt das Bestatin dar, das ebenso wie die beiden letztgenannten Inhibitoren von UMEZAwA u. Mitarb. aus Kulturlösungen von Streptomyceten isoliert wurde [29]. Die Struktur zeigt die Abb. 7 [30].

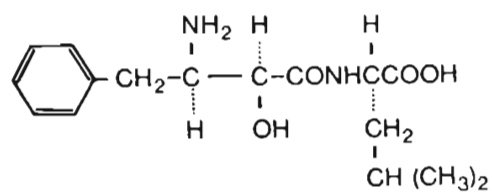

Abb. 7. Bestatin
Auch das Amastatin, ein weiterer Aminopeptidase-Inhibitor, und der Esterase-Inhibitor Esterastin, ein Mycolsäurederivat [77], stammen aus UMEZAWAs Arbeitskreis.

\section{Inhibitoren der Adrenalin- Biosynthese}

Die Biosynthese des blutdruckwirksamen Adrenalins bzw. des Noradrenalins läuft über verschiedene $Z$ wischenstufen (Abb. 8).

Die Arbeiten von Umezawa und Mitarbeitern konzentrierten sich anfangs darauf, Inhibitoren für die Enzyme (1) Tyrosin-hydroxylase und (3) Dopamin$\beta$-hydroxylase und später auch für das Enzym Catechol-O-methyl-transferase $\mathrm{zu}$ finden. Sie fanden eine Menge wirksamer Stoffe in Pilzen, Bakterien und Actinomyceten. Leider war ein Teil der gefundenen Stoffe, besonders die chinoiden Farbstoffe aus den Actinomyceten, zu wenig spezifisch.

Das Oudenon [2] aus einem Basidiomyceten hemmt die Tyrosinhydroxylase (1). Die Struktur zeigt Abb. 9.

Einige Stoffe hemmen die Dopamin$\beta$-hydroxylase (2), so die Fusarinsäure aus Fusarium oxysporum, ein schon früher als schwach antibiotisch wirkend beschriebener Stoff, der außerdem die Phe- 


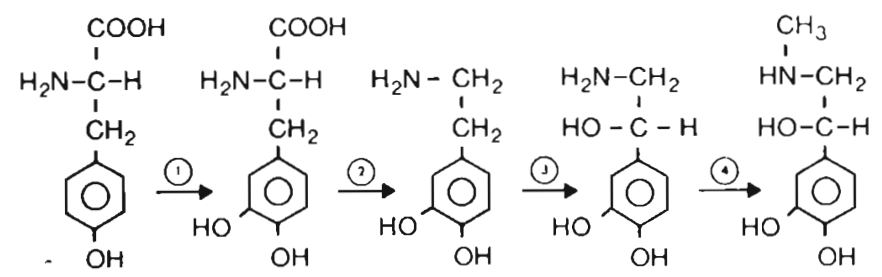

Tyrosın Dopa Dopamın Noradrenalın Adrenalin

Abb. 8. Biosyntheseweg des Adrenalins

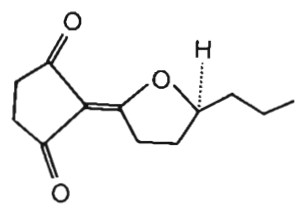

$A b b$. 9. Oudenon

noloxidase der Pflanzen hemmt. Die Fusarinsäure ist eine 5-Alkyl-picolinsäure (s. Abb. 10) [2].

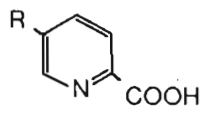

$A b b .10$. Fusarinsäure

Von den 5-Alkyl-picolinsäure-Derivaten sind die synthetisch hergestellten Butyl- bzw. Pentyl-Derivate am stärksten wirksam gegen die Dopamin- $\beta$-hydroxylase.

Aus einem Paecilomyces-Stamm wurde ein weiteres Derivat dieser Reihe isoliert, das die Dopamin- $\beta$-hydroxylase etwa doppelt so stark hemmt wie die Fusarinsäure, die Pheno-picolinsäure [31]. Die Struktur zeigt die Abb. 11.

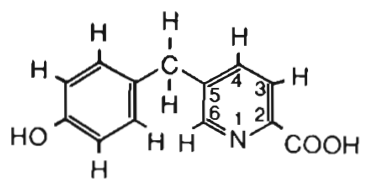

$A b b$. 11. Phenopicolinsäure

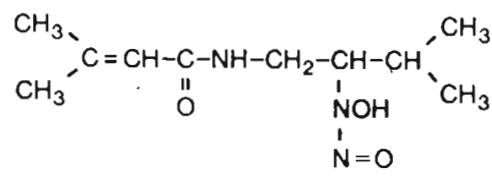

Abb. 12. Dopastin

Aus einem Pseudomonaden konnte Dopastin isoliert werden [2] (Abb. 12). Das Oosponol aus einem Basidiomyceten [2] (Abb. 13) ist ebenso wirksam gegen Dopamin- $\beta$-hydroxylase wie das Dopastin.

Abb. 13. Oosponol<smiles>O=C(CO)c1coc(=O)c2c(O)cccc12</smiles>

Von diesen Inhibitoren haben das Oudenon, die Fusarinsäure und das Dopastin eine geringe Säugertoxizität, während andere, wie z. B. das Aquayamycin, ein Dopadecarboxylase-Hemmer, in bezug auf die Toxizität ungünstiger liegen.

Auch das Enzym Catechol-O-methyltransferase wird von einer Reihe von mikrobiellen Inhibitoren gehemmt. Das Methylspinazarin (2,3,5,8-Tetra- 
hydroxy-6-methyl-1,4-naphthochinon) und das Dihydromethylspinazarin stammen aus Streptomyceten [32] (Abb. 14) und das 7-O-Methylspinochrom B (2,3, 5-Trihydroxy-7-methoxy-1,4-naphthochinon)und seine Derivate aus Fungi imperfecti [33].<smiles>Cc1cc(O)c2c(c1O)C(=O)C(O)=C(O)C2=O</smiles><smiles>C[C@H]1C(O)=C2C(=O)C(O)=C(O)C(=O)C2=C(O)C1O</smiles>

Abb. 14. Methylspinazarin, Dihydromethylspinazarin

Aus Kulturfiltraten eines Basidiomyceten Inonotus spec. wurden Kaffeesäure, d. h. die 3,4-Dihydroxyzimtsäure, und deren (+)- und (-)-Dehydrodilactone isoliert [34]. Beide Substanzen hemmen sowohl die Catechol-O-methyltransferase wie auch die Dopamin- $\beta$ hydroxylase (Abb. 15).

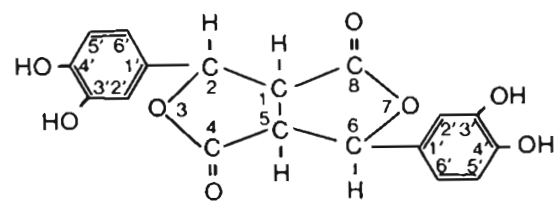

Abb. 15. Dehydrodilacton der Kaffeesäure

Auch eine Reihe von Isoflavon-Derivaten wurde aus Streptomyceten- und Aspergillen-Kulturbrühen isoliert, wobei allerdings nicht klar ist, ob es sich um de novo-Synthesen handelt oder um Umwandlungsprodukte aus pflanzlichen Nährbodenbestandteilen wie Sojamehl u. a. Die isolierten Isoflavon-Derivate waren z. T. bekannt, z. T. neu. Nur eines dieser Derivate aus Streptomyces roseolus [35] gibt eine spezifische $\mathrm{Hem}$ mung der Catechol-O-methyltransferase (Abb. 16), leider keine blutdrucksenkende Wirkung bei Ratten.<smiles>COc1ccc(-c2coc3c(O)c(OC)c(OC)cc3c2=O)cc1O</smiles>

Abb. 16. Isoflavon aus Streptomyces roseolus

Andere dieser Isoflavonderivate sind weniger spezifisch und hemmen sowohl die Catechol-O-methyltransferase, die Dopadecarboxylase und auch die $\mathrm{Hi}$ stidindecarboxylase [36, 37]. Einzelne dieser Substanzen zeigen hypotensive Effekte an hypertensiven Ratten.

\section{Weitere Hydrolasen-Inhibitoren}

UMEZAWA [2] fand in Kulturlösungen von Streptomyceten eine Hemmung von viraler Neuraminidase. Es wurde ein Inhibitor isoliert, der sich als anionisches Detergens mit breiter und unspezifischer Hemmwirkung herausstellte, das Panosialin (Abb. 17).

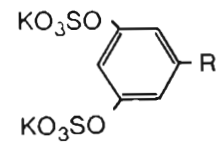

$$
\begin{aligned}
\mathrm{R}= & -\left(\mathrm{CH}_{2}\right)_{12}-\mathrm{CH}\left(\mathrm{CH}_{3}\right)_{2} \\
& -\left(\mathrm{CH}_{2}\right)_{14}-\mathrm{CH}_{3} \\
& -\left(\mathrm{CH}_{2}\right)_{13}-\mathrm{CH}\left(\mathrm{CH}_{3}\right)_{2}
\end{aligned}
$$

Abb. 17. Kaliumpanosialin

In den letzten Jahren wurde sehr intensiv nach Inhibitoren von bakteriellen $\beta$-Lactamasen gesucht [38]. Diese Inhibitoren sollen verhindern, daß Penicilline durch resistente Bakterien, die $\beta$ Lactamase produzieren, zerstört werden. Besonders die Clavulansäure [39] 


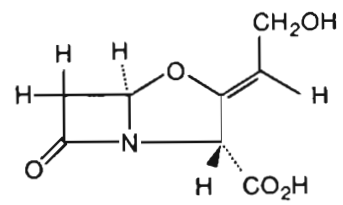

Abb. 18. Clavulansäure

(Abb. 18) steht derzeit im Vordergrund des Interesses.

UMEZAwa und Mitarbeiter konnten zeigen, daß einzelne Enzym-Inhibitoren aus Mikroorganismen auch das Tumorwachstum, wahrscheinlich durch immunstimulierende Wirkung, beeinflussen können. Einzelne dieser Inhibitoren können sich an die Oberfläche von tierischen Zellen anlagern. Neuere Inhibitoren, die in dieser Richtung genannt worden sind, sind das Formycin, ein Adenosin-deaminase-Inhibitor, und Forphenicin, ein 4-Formyl-3-hydroxy-phenylglycin und Inhibitor der alkalischen Phosphatase [40], sowie verschiedene Proteasen-Inhibitoren.

Viele Streptomyceten bilden Inhibitoren, die Glyoxalase I aus Rattenleber, aber nicht aus Hefe hemmen. Ein Stamm wurde gefunden, der die Glyoxalase I aus beiden Organismen hemmt. Die Struktur dieses Inhibitors 2-Crotonyloxymethyl - 4,5,6 - trihydroxy - cyclohexen-(2)-on zeigt die Abb. 19. Dieser Inhibitor hemmt das Wachstum von HelaZellen und Ehrlich Ascites Carcinom [41].

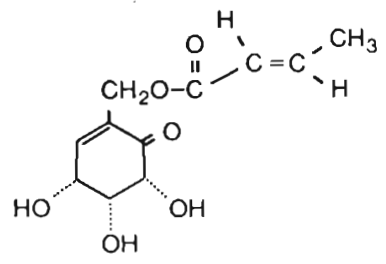

$A b b$. 19. Glyoxalase I Inhibitor
Daß man aus Mikroorganismen auch bereits bekannte Substanzen isolieren kann, zeigt die Suche von UmEzAwa nach Monoamino-oxidase-Inhibitoren. Es wurden je 500 Actinomyceten- und Basidiomyceten-Kulturen auf Hemmung von Serotonin- bzw. BenzylaminOxidation mit Monoamino-oxidase untersucht. Alle 4 gefundenen Wirksubstanzen waren bereits bekannt [42].

Ebenso bekannt war das Reticulol, ein 6,8-Dihydroxy-7-methoxy-3-methylisocumarin, das von mehreren $\mathrm{Ar}$ beitskreisen aus Streptomyceten-Kulturfiltraten isoliert wurde, dessen inhibitorische Wirkung auf c-AMP-Phosphodiesterase aber erst gefunden wurde, nachdem es erneut nach einem Screening auf Stämme, deren Kulturfiltrate cAMP-Phosphodiesterasen hemmen, isoliert wurde [43].

\section{Glycosid-Hydrolasen-Inhibitoren}

\section{Problemstellung}

Wir gingen bei unseren Versuchen nach Vorstellungen von Puls u. a. von rein theoretischen Uberlegungen aus [44]. Die Kohlenhydrate unserer Nahrung bestehen überwiegend aus Stärke und Saccharose. Ihr Verzehr bewirkt u. a.:

- bei Diabetikern eine besonders ausgeprägte postprandiale Hyperglykämie, - bei Adipösen eine überschießende Sekretion von Insulin, das die Speicherung von Neutralfett im Fettgewebe fördert,

- bei Patienten mit Hyperlipoproteinämie eine Steigerung der Synthese von triglycerid-reichen Lipoproteinen

(Abb. 20). 


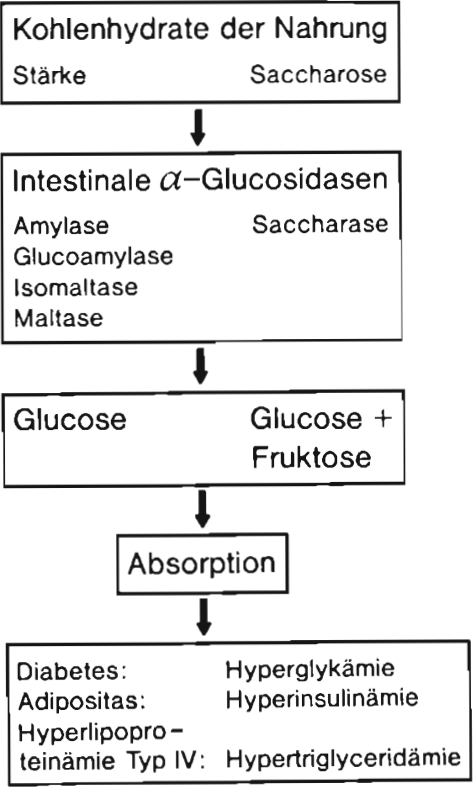

Abb. 20. Kohlenhydrate der Nahrung

Die unerwünschten Folgen des Kohlenhydratverzehrs kann man abschwächen bzw. vermeiden:

1. durch eine Beschränkung der Kohlenhydratmenge,

2. durch eine Verteilung der Kohlenhydratzufuhr in möglichst kleine Portionen,

3. durch eine Verzögerung/Verminderung der Kohlenhydratabsorption,

4. durch eine Verzögerung des Abbaus der Nahrungskohlenhydrate mit Hil$\mathrm{fe}$ von Glycosid-Hydrolasen-Inhibitoren.

\section{Erste Versuche mit pflanzlichen Amylase-Inbibitoren}

Außer einigen unspezifischen z.T. tanninartigen pflanzlichen Amylase-Inhibitoren wurden auch spezifische $a$ Amylase-Inhibitoren beschrieben, die in Kolokasienknollen [45], Bohnen [46] und Getreide [47] vorkommen und Pro- teincharakter besitzen. Wir isolierten aus Weizensamen ein vor allem gegen $\alpha$-Amylase aus Schweinepankreas wirksames Inhibitorpräparat [48].

Mit diesem Inhibitor konnten Puls und Keup [49] zeigen, daß die Verweildauer der Stärke im Magen und Dünndarm von Ratten bei gleichzeitiger oraler Inhibitor-Applikation signifikant verlängert wird. Außerdem konnte sowohl bei Ratten als auch an gesunden Probanden eine signifikante Senkung der postprandialen Hyperglykämie und Hyperinsulinämie nach gleichzeitiger oraler Gabe von Stärke und Inhibitor nachgewiesen werden.

Für eine therapeutische Anwendung war dieser Weizen-Inhibitor aufgrund seiner Proteinnatur nicht geeignet, da er von den Proteasen des Verdauungstraktes zu schnell inaktiviert wurde. Die Suche nach proteolytisch nicht abbaubaren Inhibitoren wurde deshalb durch ein Screening mikrobieller Kulturfiltrate begonnen.

Vorkommen von Glycosid-HydrolasenInbibitoren in Mikroorganismen

Wir fanden in praktisch allen Gattungen der Ordnung Actinomycetales, von denen eine gewisse Anzahl von Stämmen zur Verfügung stand, auffallend häufig deutliche Hemmwirkung gegen Glycosid-Hydrolasen, vor allem gegen Glycosid-Hydrolasen des Säugetierverdauungstraktes, vor allem auch bei Stämmen der Familie Actinoplana$c e a e^{1}$, die bis dahin noch relativ wenig auf Sekundärstoffbildung untersucht worden waren.

1 Die Actinoplanaceae wurden von A. HENSSEN und D. SCHÄFER, Fachbereich Biologie, Botanik, Universität Marburg, isoliert. 


\section{Tabelle III}

Vorkommen von Amylase- und Saccharase-Inhibitionen bei Stämmen verschiedener Gattungen

\begin{tabular}{lllr}
\hline Gattung & $\begin{array}{l}\text { Anzahl der } \\
\text { getesteten } \\
\text { Stämme }\end{array}$ & $\begin{array}{l}\text { gegen Schweinepankreas- } \\
\text { Amylase wirksame Stämme }\end{array}$ & $\begin{array}{l}\text { gegen Schweinedünndarm- } \\
\text { Saccharase wirksame Stämme }\end{array}$ \\
\hline Streptomyces & 85 & 3 & 2 \\
Actinoplanes & 220 & 43 & 24 \\
Streptosporangium & 161 & 18 & 24 \\
\hline
\end{tabular}

Die Tab. III zeigt einen kleinen Ausschnitt aus diesen Versuchen. Sie gibt Häufigkeit der gegen Schweine-Pankreas-Amylase und Schweine-Dünndarm-Saccharase wirksamen Stämme bei einzelnen Gattungen wieder, so wie wir sie nach unserem ersten ScreeningDurchgang gefunden haben (Beschreibung der Teste [3]).

Manche Stämme waren nur gegen ein Enzym wirksam, manche gegen mehrere Enzyme. Wir reicherten bei einer großen Anzahl von Stämmen die Wirkstoffe gegen $a$-Amylase aus Schweinepankreas an. Bei diesen Anreicherungen konnten wir 2 Gruppen von Wirkstoffen unterscheiden.

1. Die eine Gruppe ist hitzelabil und nicht oder kaum dialysierbar. Diese Inhibitoren sind durch Trypsin, Harnstoff oder $\beta$-Mercaptoäthanol inaktivierbar.

Die besten dieser Anreicherungen zeigten Hemmaktivitäten von 80 AIE $/ \mathrm{ml}^{2}$ [3]. Tierversuche mit einzelnen Anreicherungspräparaten zeigten Effekte wie der oben genannte Weizenamylaseinhibitor.

Bei diesen Stoffen handelt es sich um Inhibitoren mit Peptidcharakter.

2. Besonders interessant erschien uns eine 2. Gruppe von Inhibitoren, die bei neutralem $\mathrm{pH}$ hitzestabil, säurestabil ( $\mathrm{pH} 2)$, alkalistabil ( $\mathrm{pH} 12)$ und langsam dialysierbar sind. Diese Inhibitoren werden durch Trypsin und Pepsin, Harnstoff und Mercaptoäthanol nicht inaktiviert.

Einzelne Stämme, die gleichzeitig Hemmwirkung gegen Säugetier-Amylase, -Maltase und -Saccharase zeigten und deren Inhibitoren Oligosaccharidcharakter haben, wurden von uns bevorzugt bearbeitet, insbesondere die Actinoplanes-Stämme SE 50, SE 82 und SB 18.

Alle drei Stämme sind aufgrund ihrer Sporophorstruktur und der Beweglichkeit der Sporen eindeutig der Gattung Actinoplanes zuzuordnen.

Das Vorkommen von $\alpha$-Amylase-Inhibitoren wird in den letzten Jahren durch verschiedene Publikationen bestätigt. Einzelne der in der Literatur beschriebenen Inhibitoren gehören offensichtlich mehr zur Gruppe 1, andere mehr zur Gruppe 2. Eine Übersicht gibt Tab. IV.

Neben diesen vorwiegend gegen $\alpha$ Glycosidasen wirksamen Stoffen kommen in Mikroorganismen auch Stoffe vor, die vorwiegend gegen $\beta$-Glycosidasen wirken, wie das Nojirimycin, das als schwach wirksames Antibiotikum 


\section{Tabelle IV}

$\alpha$-Amylase-Inhibitoren aus Mikroorganismen

\begin{tabular}{|c|c|c|}
\hline Autoren & Organismus & Struktur bzw. Hydrolyseprodukte \\
\hline $\begin{array}{l}\text { FromMER, W. } \\
\text { PULS, W. } \\
\text { SCH ̈̈FER, D. } \\
\text { SCHMIDT, D. } \\
\text { [3] (1970) }\end{array}$ & $\begin{array}{l}\text { Actinomyceten } \\
\text { verschiedener Gattungen }\end{array}$ & Oligosaccharide und Proteine \\
\hline $\begin{array}{l}\text { UEDA, S. } \\
\text { KoBA, Y. }\end{array}$ & $\begin{array}{l}\text { Streptomyces } \\
\text { flavochromogenes }\end{array}$ & Glutaminsäure, Asparaginsäure \\
\hline $\begin{array}{l}{[50](1971)} \\
\text { Goто, H. }\end{array}$ & & Serin, Alanin \\
\hline INUKaI, T. & Streptomyces & Asparaginsäure \\
\hline $\begin{array}{l}\text { Amano, M. } \\
\text { [51] (1973) }\end{array}$ & fradiae & $\begin{array}{l}\text { Asparagin } \\
\text { u. a. Aminosäuren }\end{array}$ \\
\hline $\begin{array}{l}\text { Murao, S. } \\
\text { [52] (1974) }\end{array}$ & $\begin{array}{l}\text { Streptomyces diastaticus } \\
\text { v. amylostaticus }\end{array}$ & Glucose $($ ca. $1 \% \mathrm{~N})$ \\
\hline $\begin{array}{l}\text { UEdA, K. } \\
\text { Gochyo, S. } \\
\text { [53] (1974) }\end{array}$ & Streptomyces spec. & Glucose \\
\hline $\begin{array}{l}\text { Belloc, A. } \\
\text { Florent, J. } \\
\text { Lunel, J. } \\
\text { Mancy, D. } \\
\text { Pulla, J. C. } \\
\text { [54] (1976) }\end{array}$ & Streptomyces calidus & $\begin{array}{l}\text { Lysin } \\
\text { Glucose }\end{array}$ \\
\hline $\begin{array}{l}\text { Koba, Y. } \\
\text { NaJima, M. } \\
\text { Ueda, S. } \\
{[55](1976)}\end{array}$ & Streptomyces & $\begin{array}{l}\text { Aminosäuren } \\
\text { Glucose }\end{array}$ \\
\hline $\begin{array}{l}\text { Oeding, V. } \\
\text { PfaAf, W. } \\
\text { VerTEsy, L. } \\
\text { WeidemÜLler, H.-L. } \\
\text { [74] (1977) }\end{array}$ & Streptomyces & Aminosäuren \\
\hline
\end{tabular}

entdeckt wurde [56] und dessen enzyminhibitorische Wirkung später beschrieben wurde $[57,58]$ (Abb. 21).

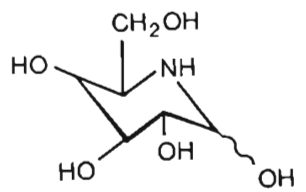

$A b b$. 21. Nojirimycin
Das 1-Desoxynojirimycin wird neben anderen Glucosid-Hydrolasen-Inhibitoren auch von Stämmen der Gattung $B a$ cillus gebildet [75].

Pyridindolol, ein spezifischer Inhibitor von neutraler $\beta$-Galaktosidase aus Rinderleber, wurde von UMEZAwA et al. $[59,60] 1975$ beschrieben (Abb. 22).

Ein etwas breiter wirkender $\beta$-Galaktosidase-Inhibitor, der sowohl saure wie 


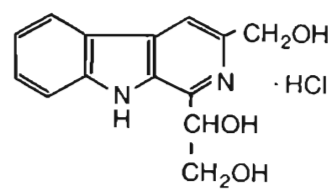

Abb. 22. Pyridindolol

auch neutrale $\beta$-Galaktosidase aus Rinderleber hemmt, wurde als IsoflavonRhamnosid identifiziert [61].

Weitere Inhibitoren, die Sialidase (N-Acetyl-neuraminat-glycohydrolase) hemmen, die Siastatine A u. B, wurden ebenfalls aus Kulturfiltraten von Streptomyceten isoliert [62].

\section{Herstellung von Amylase-Inbibitoren}

In ersten Kulturversuchen mit den oben aufgeführten Actinoplanes-Stämmen fanden wir, daß sich die Inhibition von Amylase, Maltase und Saccharase nicht gleichmäßig mit den Kulturbedingungen verändert. Wir schlossen daraus, daß die Stämme Wirkstoffgemische produzieren. Da Stärke bei den üblichen mitteleuropäischen Eßgewohnheiten der Hauptanteil der Kohlenhydratzufuhr ist, begannen wir, die Fermentationsbedingungen im Hinblick auf optimale Amylase-Inhibitor-Produktion zu variieren.

Es zeigte sich bei den zuvor genannten drei Stämmen, daß die Art der Kohlenstoffernährung einen entscheidenden Einfluß auf die Produktion der AmylaseInhibitor-Aktivität hat. Mit Mono- und Disacchariden als alleiniger C-Quelle erhielten wir nur mäßige Titer an Amylase-Inhibition. Gibt man Stärke zu einer Nährlösung zu, so steigt die Ausbeute beträchtlich [63, 64], in gewissen Grenzen proportional mit dem Stärkezusatz.

Bei 5,5-6\% Stärkezusatz wird ein Optimum erreicht, bei dem man mit dem besonders aktiven Stamm SE 50/13 je Liter Kulturbrühe 10-13 g an Produkt BAY e 4609 (s. u.) erhält.

Die übrigen Bestandteile der Nährlösung können ohne Beeinträchtigung der Ausbeute in erheblichen Grenzen variiert werden.

Ausgehend von diesen Fermentationen entwickelten wir ein einfaches Herstellungsverfahren für ein etwa $20 \%$ iges $\alpha$-Amylase-Inhibitor-Standardpräparat, das für tierexperimentelle Zwecke und, wie sich herausstellte, aufgrund seiner toxikologischen Unbedenklichkeit auch für Probandenstudien geeignet ist.

Dieses Präparat, BAY e 4609 , besteht neben Dextrinen aus der Nährlösung aus einem Gemisch von $\alpha$-AmylaseInhibitoren. Es hat eine spezifische Amylase-Inhibitor-Aktivität von $3 \times 10^{6}$ $\mathrm{AIE} / \mathrm{g}^{2}$ nach FIP [65] und wirkt sowohl gegen Säugetier-Pankreas- und Speichel- $\alpha$-Amylasen als auch gegen pilzliche Gluco-Amylasen.

Hemmkurven für einzelne Amylasen zeigt die Abb. 23.

Bei der Analyse der Oligomerenverteilung [64] erreichten wir durch Gelchromatographie an Bio-Gel P-6, 200400 mesh (Säule $28 \times 2000 \mathrm{~mm}, 100 \mathrm{mg}$ $\mathrm{BAY}$ e $4609, \mathrm{H}_{2} \mathrm{O}, 65^{\circ} \mathrm{C}$ ) eine Auftrennung in 2 hemmaktive Anteile unterschiedlicher Molekulargewichtsverteilung. Das Eluat wurde refraktometrisch, konduktometrisch, durch Anthrontest und durch $\alpha$-Amylase-Hemmung geprüft (Abb. 24).

Auffallend ist die diskontinuierliche Verteilung. Ein höhermolekulares aktives Gemisch (MG um 6000) erscheint

${ }^{2} \mathrm{AIE}=$ Amylase-Inhibitor-Einheit [3]. 


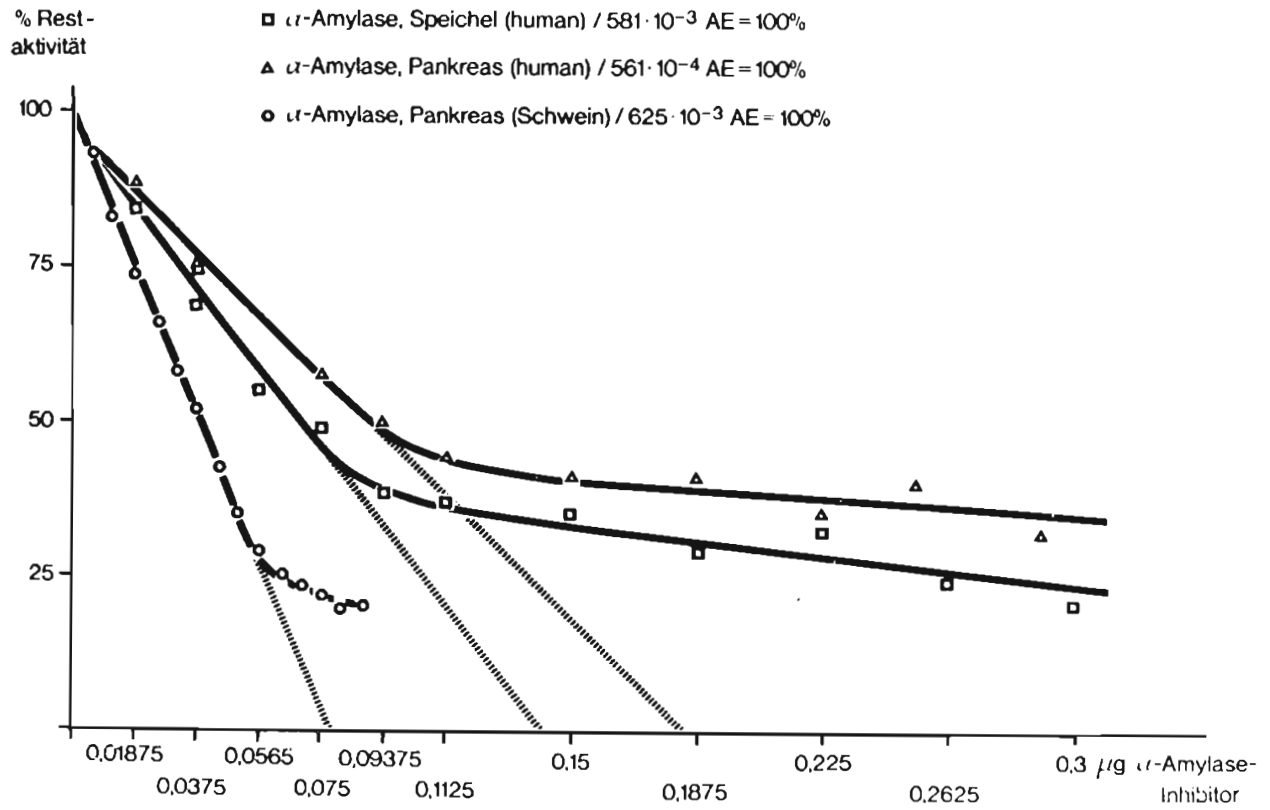

Abb. 23. $\alpha$-Amylase-Hemmung durch $\alpha$-Amylase-Inhibitor BAYe 4609.

10 Minuten Vorinkubation bei $35^{\circ}$ und $\mathrm{pH} 6,9$.

$\mathrm{AE}=$ Amylaseeinheiten nach [3].

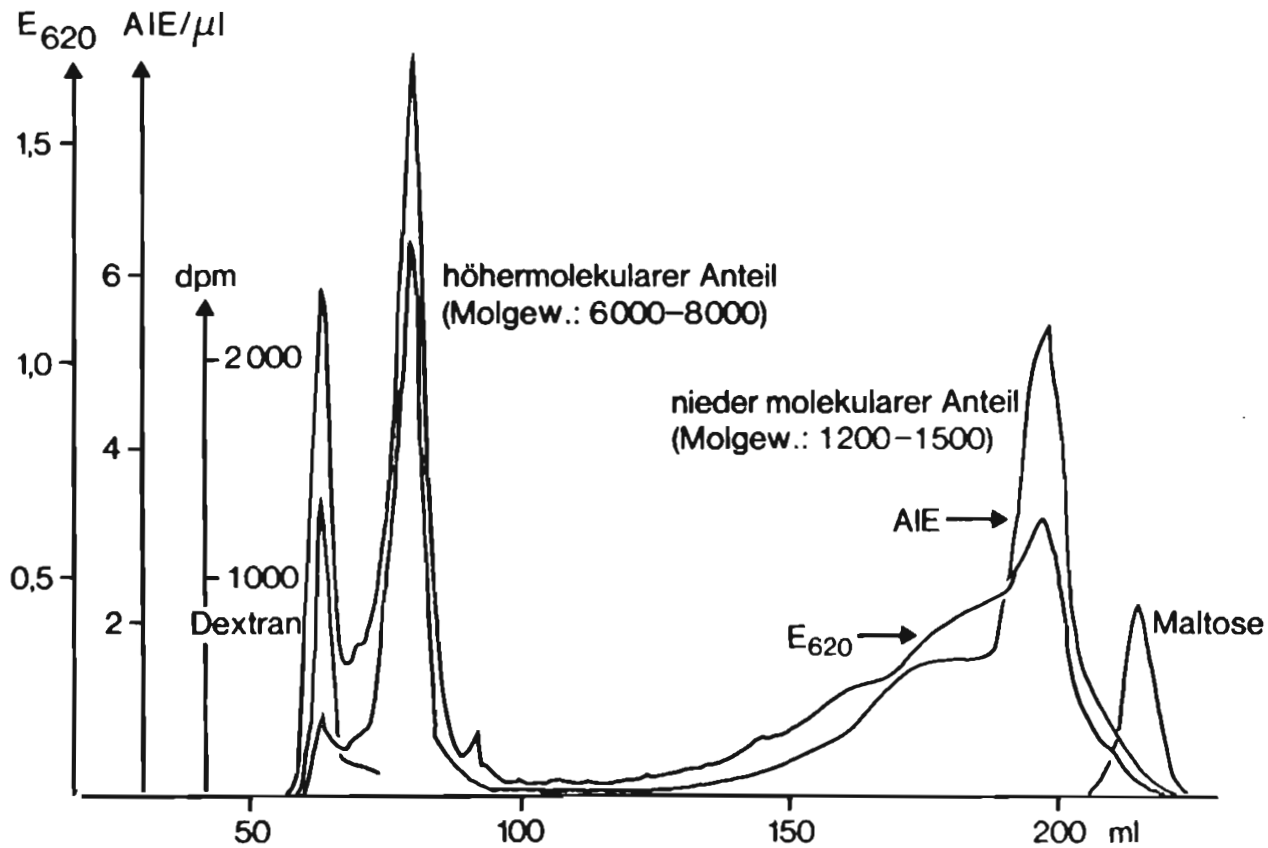

Abb. 24. Gelchromatographie des $\alpha$-Amylase-Inhibitors BAYe 4609 an Bio-Gel P-6. 
unmittelbar nach dem void volume. Rechromatographie des niedermolekularen peaks an Bio-Gel P-2 zeigt, daß das scharfe Maximum beim Elutionsvolumen einer Maltononaose liegt, also ein Molekulargewicht um 1500 haben dürfte.

Durch Rechromatographie an Dowex $50 \mathrm{~W}$ gelang es, eine von inerten Kohlenhydraten weitgehend freie Präparation des niedermolekularen Anteils zu erhalten mit einer spezifischen Aktivität von $15 \times 10^{6} \mathrm{AIE} / \mathrm{g}$ (nach FIP).

Herstellung von Inbibitoren der intestinalen Disaccharidasen

Die Kulturlösungen der Stämme SE 50, SE 82 und SB 18 hemmen nicht nur $\alpha$-Amylasen sondern auch intestinale Maltasen, Glucoamylase und Saccharasen. Die Tatsache, daß das Verhältnis AIE zu SIE ${ }^{3}$ je nach Kulturlösung schwankte, gab zu der Vermutung Anlaß, daß in diesen Kulturbrühen je nach Kulturbedingung andere Inhibitorgemische gebildet werden.

${ }^{3} \mathrm{SIE}=$ Saccharase-Inhibitor-Einheit [3].
Wir haben nun versucht, durch Variation der Kulturbedingungen und durch Stammselektion Bedingungen zu erarbeiten, unter denen wir möglichst selektiv Saccharase-inhibitorische Aktivitäten erhalten.

Die Tab. V soll einen Eindruck geben, wie unterschiedliche Stämme reagieren können. Die Stämme SE 50/13 und SE 50/110 sind durch einfache Auslese ohne Mutagenbehandlung aus dem Wildstamm SE 50 entstanden [63, 64].

Maltose erwies sich als besonders günstige Kohlenstoffquelle zur Erzeugung von hohen SIE-Titern, aber auch zusammen mit Glucose zur Gewinnung eines günstigen SIE/AIE-Verhältnisses (Tab. VI).

\section{Strukturaufklärung der $\alpha$-Glucosidasen-} Inbibitoren

Wir konnten zeigen, daß die aus den Stämmen SE 50, SE 82 und SB 18 gewonnenen Amylase- und Saccharaseinhibitoren strukturell verwandt sind und aus einem stickstoffhaltigen core sowie einer unterschiedlichen Anzahl von D-Glucoseeinheiten aufgebaut sind. So

\section{Tabelle V}

Saccharase- und Amylase-Inhibitor-Titer bei verschiedenen Stämmen

AIE/ml nach 3 Tagen SIE/ml nach 3 Tagen

Nährlösung Y 1

Stamm SE 50

Stamm SE 50/13

Stamm SE 50/110

Nährlösung S 1

Stamm SE 50

Stamm SE 50/13

Stamm SE 50/110
37000

109000

53000

$\begin{array}{rl}580 & 25 \\ 1500 & 15 \\ 760 & 58\end{array}$


Tabelle VI

Kohlenstoff-Ernährung und Inhibitorproduktion (Stamm SE 50)

\begin{tabular}{lccc}
\hline Kohlenstoff quelle & \multicolumn{2}{c}{ Titer nach 4 Tagen } \\
& SIE/L & AIE/ml & SIE \\
& & & AIE $\times 10^{-3}$ \\
\hline Glycerin 3\% & 160 & 100 & 1,6 \\
Galactose 3\% & 1100 & 100 & 11 \\
Maltose 3\% & 5500 & 1000 & 5,5 \\
Zellobiose 3\% & 1300 & 220 & 6 \\
Glycerin $1 \%+$ Glucose 2\% & 2700 & 120 & 22 \\
Galactose $1 \%+$ Glucose 2\% & 2700 & 120 & 22 \\
Maltose $1 \%+$ Glucose 2\% & 6600 & 200 & 33 \\
Zellobiose $1 \%+$ Glucose 2\% & 2200 & 105 & 21 \\
\hline
\end{tabular}

führt beispielsweise die milde Hydrostarker Saccharase-inhibitorischer Aktilyse des Amylase-Inhibitorpräparates vität (Abb. 25) [67]. BAY e 4609 mit 0,5 N HCl unter Ab- Die strukturanalytischen Untersuspaltung von $\mathrm{D}-\mathrm{Glucoseeinheiten} \mathrm{zu} \mathrm{N}^{-}$chungen wurden vor allem an dem ferhaltigen Partialhydrolyseprodukten mit mentativ hergestellten Saccharaseinhibi-

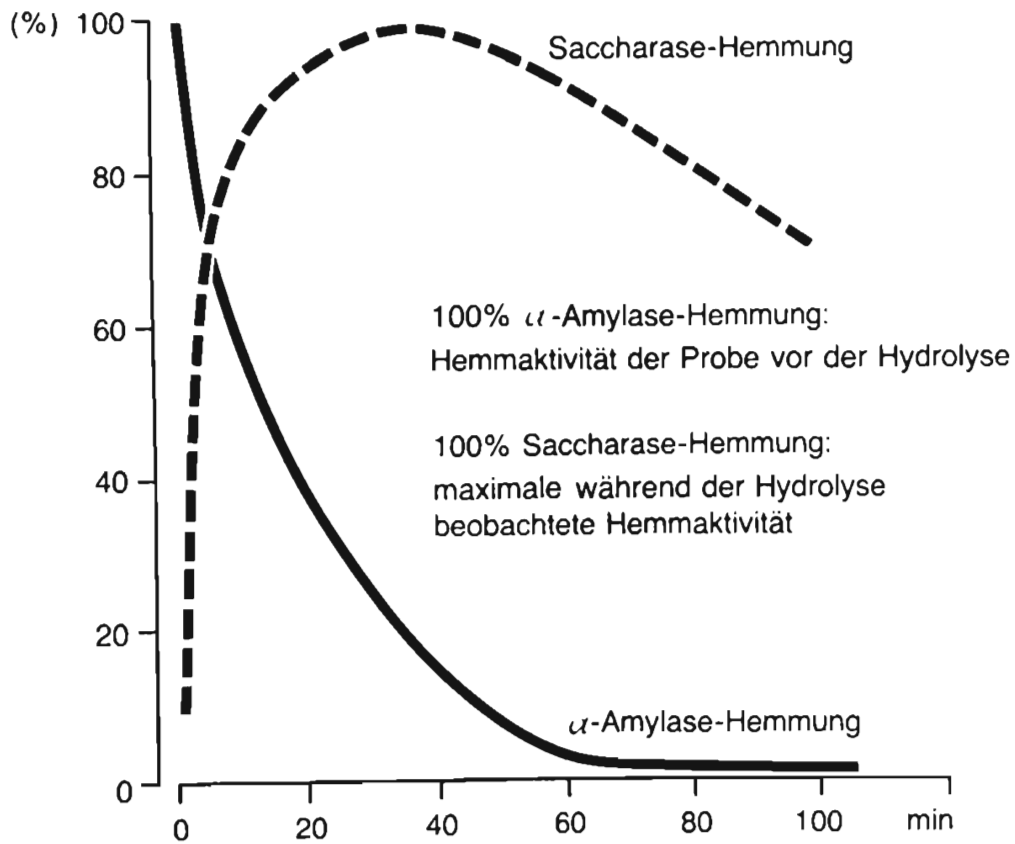

Abb. 25. Hydrolyse des $\alpha$-Amylase-Inhibitors BAYe 4609 mit $0,5 \mathrm{~N} \mathrm{HCl}$ bei $100^{\circ} \mathrm{C}$. 
tor BAY g 5421 durchgeführt. Für diese Verbindung konnte die in Abb. 26 für Komponente 3 angegebene Struktur ermittelt werden. Der Strukturbeweis stützt sich vor allem auf folgende $\mathrm{Ab}$ baureaktionen $[66,68]$.

Die Hydrogenolyse mit $\mathrm{Pt} / \mathrm{H}_{2}$ spaltet das Molekül in das literaturbekannte Validatol [69] und ein stickstoffhaltiges Trisaccharid. Letzteres wird mit Acetanhydrid/Schwefelsäure weiter zu dem bekannten Aminozuckerderivat 1,2,3Tri-O-acetyl-4-acetamido-4,6-didesoxy$\alpha$-D-glucopyranose [70] und 2 Molekülen D-Glucosepentaacetat abgebaut. Durch Hydrolyse mit starken Mineralsäuren lassen sich aus BAY g 5421 sukzessiv beide Glucoseeinheiten entfernen. Das verbleibende stickstoffhaltige core
(Cyclitoleinheit + Aminozucker) ist unter den Reaktionsbedingungen aber nicht stabil und kondensiert zu einer tricyclischen Verbindung (Abb. 27), deren Struktur durch Abbaureaktionen und spektroskopische Untersuchungen bewiesen wurde.

In unseren Inhibitoren ist die Cyclitoleinheit jedoch nicht immer endständig angeordnet. Für einige glucosereichere Inhibitoren konnte nämlich gezeigt werden, daß sich das $\mathrm{N}$-haltige core inmitten einer Kette $a$-1-4-verknüpfter D-Glucoseeinheiten befindet. Somit läßt sich die Struktur unserer Inhibitoren allgemein durch die in Abb. 28 wiedergegebene Formel beschreiben.

Die niederen Glieder dieser homologen Reihe zeichnen sich vor allem durch
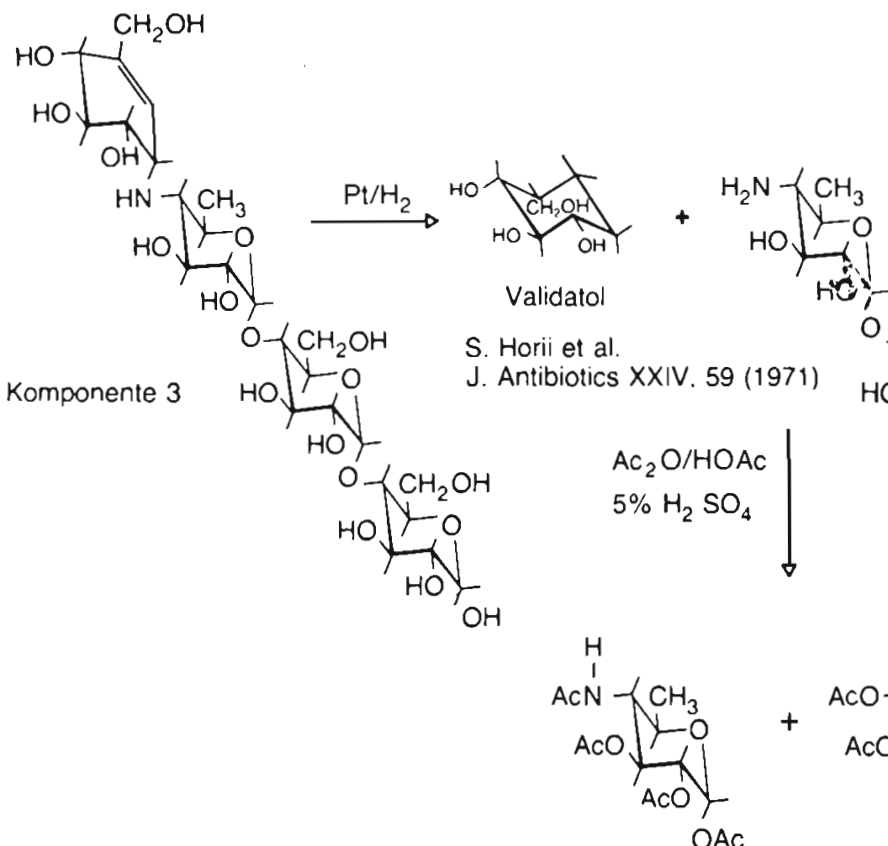

Validatol

Horii et al.

Antibiotics XXIV. 59 (1971)

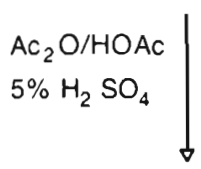

$\mathrm{O}-\mathrm{CH}_{2} \mathrm{OH}$

HOY

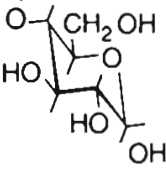

OAC

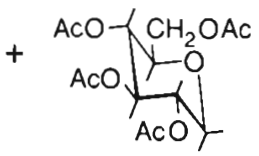

OAC

C. L. Stevens et al.

J. Org. Chem. 31, 2822 (1966)

Abb. 26. Abbaureaktionen Komponente 3 . 


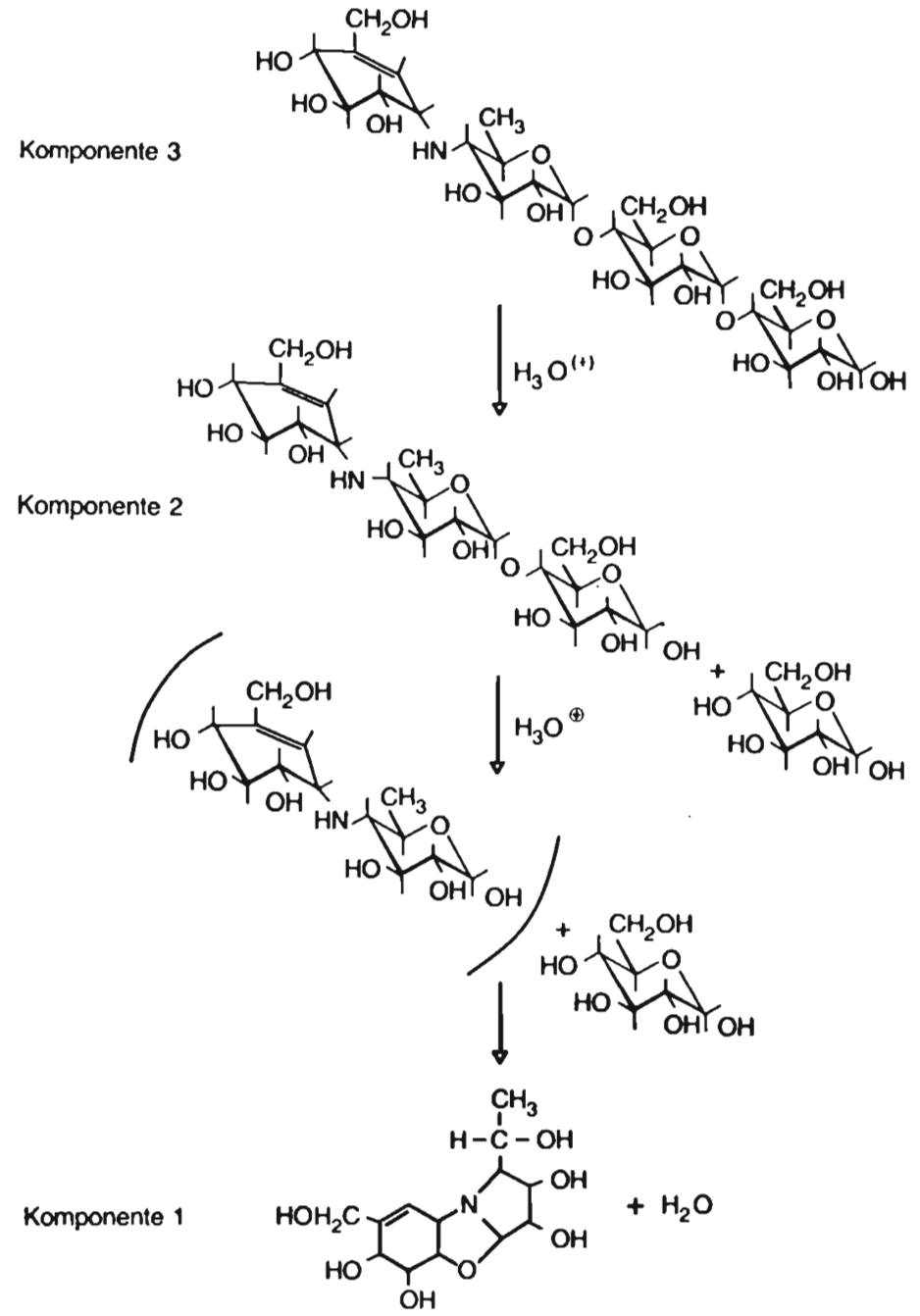

Abb. 27. Hydrolyse von Komponente 3.

die Hemmung der intestinalen Disaccharidasen, die höheren Glieder durch die Hemmung der $\alpha$-Amylase aus.

Direkte fermentative Herstellung der niederen Oligomeren

Schon bei der Herstellung der Amylase-Inhibitoren war aufgefallen, daß die Art des gebildeten Inhibitors stark von der Kohlenhydraternährung wäh- rend der Fermentation abhängt. Daneben konnten wir eine Stammlinie entwickeln, die bevorzugt die niederen Homologen bildet. Maltose wurde als besonders günstige Kohlenstoffquelle für die Herstellung von niederen Homologen erkannt. Die Ausnutzung dieser Beobachtungen führte im Verlauf der weiteren Arbeiten zu mehreren Verfahren, nach denen wir heute selektiv einzelne 


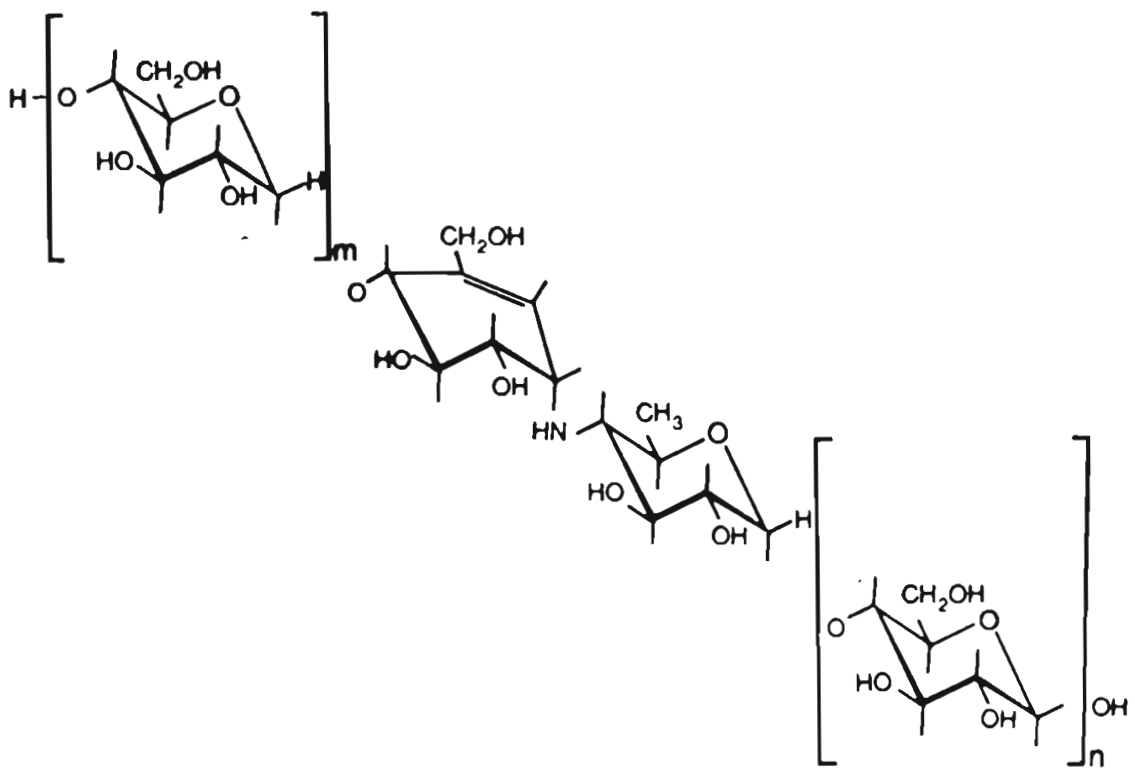

Abb. 28. Allgemeine Strukturformel der $\alpha$-Glucosidase-Inhibitoren.

Komponenten oder bestimmte Komponentengruppen herstellen können [63, 64]. Damit hatten wir auch die Möglichkeit in der Hand, größere Mengen an einzelnen Gliedern der homologen Reihe für tierexperimentelle Prüfungen zu erhalten.

\section{a) Selektive Herstellung von Kompo- nente 2 ( $A b b .28, m=0, n=1$ )}

Die Komponente 2 tritt dann vorwiegend auf, wenn wir nur mit Glucose als C-Quelle arbeiten. Durch Einstellung eines optimalen $\mathrm{C}: \mathrm{N}-$ Verhältnisses unter Variation von Glucose und Stickstoffquellen in der Nährlösung konnten wir die Bildung der höheren Komponenten weitgehend unterdrücken.

Wir erhielten mit dem Stamm SE 50/ 110 Kulturfiltrate mit etwa $400 \mathrm{mg}$ Komponente 2 je Liter. b) Selektive Herstellung von Komponente 3 ( $A b b .28, m=0, n=2$ )

Mit Maltose als Kohlenstoffquelle wird vorwiegend Komponente 3 gebildet. In einer Nährlösung mit 7,5\% Maltzin (Malzextrakt-Trockenpulver der Firma Diamalt AG), einer passenden Stickstoffquelle und Puffersubstanzen erhält man mit dem Stamm SE 50/ $11070000-80000$ SIE/Ltr. Dies entspricht bei einer spezifischen Aktivität von Komponente 3 von $77 \mathrm{SIE} / \mathrm{mg}$ etwa $1 \mathrm{~g} / \mathrm{Ltr}$. In dieser Aktivität sind über $80 \%$ an Komponente 3 enthalten. Die Restaktivität entfällt auf die Komponenten 4, 5 und Komponente 2.

c) Selektive Herstellung von höher molekularen Komponenten

Bei Zusatz von kleinen Mengen von Stärke zu glucosehaltigen Nährlösun- 


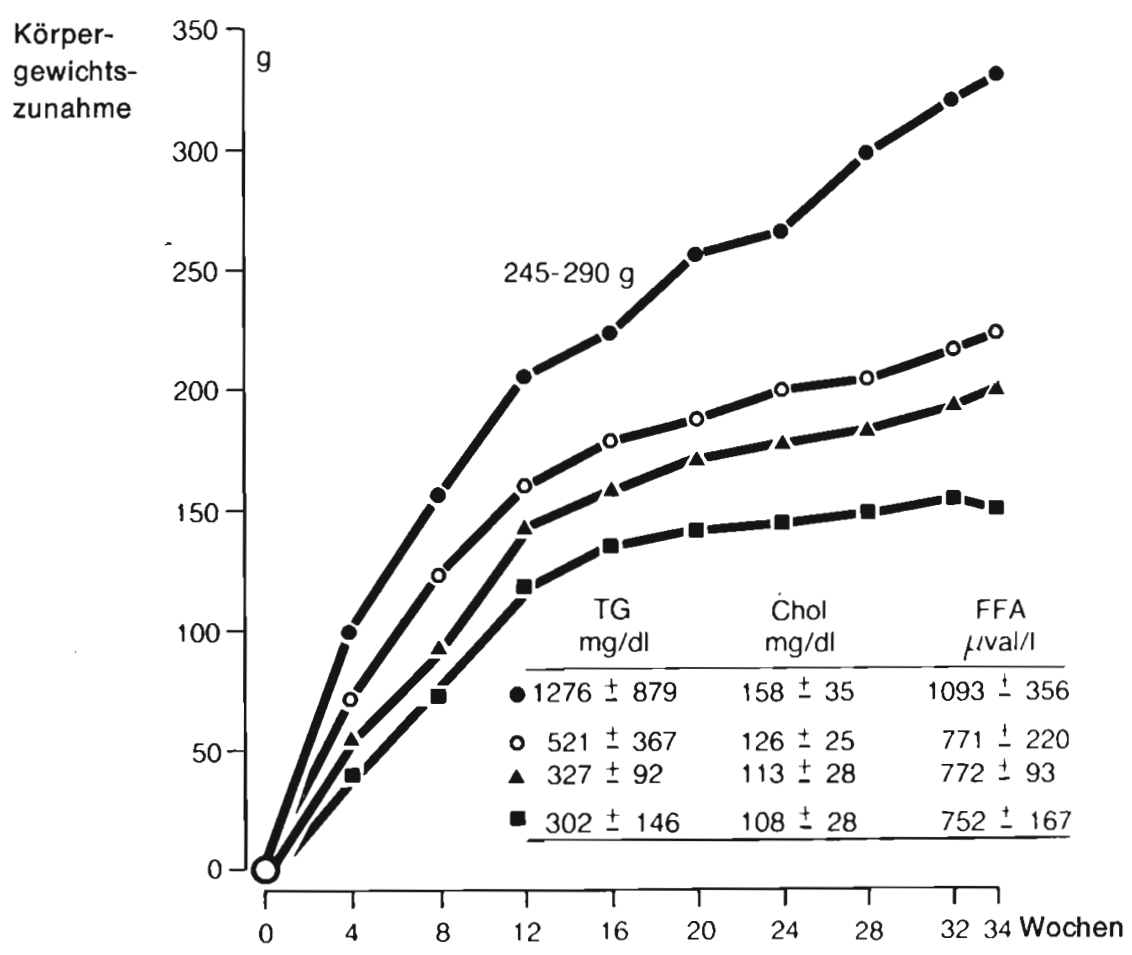

Abb. 29. Wirkung des Glucosidase-Inhibitors BAYg 5421 auf die Körpergewichtszunahme, den Serumtrigylceridspiegel (TG), den Serumcholesterinspiegel (Chol) und die freien Fettsäuren (FFA) im Blut von genetisch fettsüchtigen und hyperlipämischen Ratten (W. Puls, U. Keup, H. P. Krause, G. Thomas, F. Hoffmeister 1977).

-O Kontrolle

$\mathrm{O}-\mathrm{O}=$ Bay g $5421,20 \mathrm{mg} / 100 \mathrm{~g}$ Futter

Anfangsgewicht $=245-290 \mathrm{~g}$

gen werden bevorzugt die mittleren Glieder der homologen Reihe gebildet. Ändert man das Glucose:Stärke-Verhältnis, so kann man den Anteil der einzelnen Komponenten stark variieren. Außerdem ändert sich das Komponentenverhältnis im Laufe der Fermentation.

Man erhält je nach dem Konzentrationsverhältnis Glucose:Stärke Fermentationen, die im jungen Zustand vor-
$\boldsymbol{\Delta}-\boldsymbol{\Delta}=$ Bay g $5421,40 \mathrm{mg} / 100 \mathrm{~g}$ Futter

D = Bay g $5421,80 \mathrm{mg} / 100 \mathrm{~g}$ Futter

\section{Pharmakologische Untersuchungen}

Uber die ersten pharmakologischen Untersuchungen mit diesen Substanzen berichten Puls et al. [71, 72, 73].

Sowohl Ratten wie auch gesunde Pro- 
banden reagieren bei einer Zuführung des Amylase-Inhibitors BAY e $4609 \mathrm{zu}-$ sammen mit stärkehaltiger Nahrung mit einer deutlichen dosisabhängigen Verminderung des Blutglucose- und Seruminsulinanstiegs. Eine entsprechende Wirkung zeigt die Zuführung des Saccharase-Inhibitors BAY g 5421 zusammen mit Saccharose und überraschenderweise auch bei Zuführung zusammen mit Stärke, obwohl BAY g 5421 den Stärkeabbau durch Amylase in vitro nur schwach hemmt.

Den Einfluß von BAY g 5421 auf die Körpergewichtszunahme, die Serumtriglyceride, das Serumcholesterin und die freien Fettsäuren bei genetisch adipösen Ratten zeigt die Abb. 29 [73].

Mit diesen und ähnlichen Versuchen konnte gezeigt werden, daß sich mit der Verzögerung des Kohlenhydratabbaus durch Glycosid-Hydrolasen-Inhibitoren unerwünschte Folgen des Kohlenhydratverzehrs vermindern lassen.

Diese Ausführungen sollen eine Übersicht über die erst in den letzten Jahren aufgefundenen Enzym-Inhibitoren aus Mikroorganismen geben.

Diese Ubersicht soll zeigen, daß die Mikroorganismen in der Lage sind, weit mehr als nur Antibiotika zu bilden, vielmehr daß die Mikroorganismen ein weites Reservoir von neuen Stoffen darstellen.

Dazu ist es notwendig, neue Teste aufzubauen. Da der Aufwand für den Aufbau der Teste, für die Auffindung von Stoffen und für die Strukturermittlungen beträchtlich ist, sollte man betont versuchen, solche Testmodelle aufzubauen, die einen Bezug zur praktischen Anwendung haben.

\section{Literatur}

1. Berdy, J., in Perlman, P. (ed.): Advances in Applied Microbiology 18, 309 (1974). New York, Academic Press.

2. Umezawa, H.: Enzyme Inhibitors of Microbial Origin, Baltimore, London, Tokyo 1972, University Park Press.

3. Frommer, W., W. Puls, D. Schäfer u. D. Schmidt: DOS 2064092 v. 20. 7. 1972, Prioritätsdatum 28. 12. 1970, Inhibitoren für Glycosidhydrolasen aus Actinomyceten.

4. Kassel, B.: Methods in Enzymology 19, 839 (1970).

5. Hoeyen, T., A. Skulberg: Nature (Lond.) 195, 922 (1962).

6. Brecher, A. S. u. R. D. Pugatsch: Experientia 25, 251 (1969).

7. Fossum, K.: Acta path. microbiol. Scand., Sekt. B, 78, 755 (1970).

8. Yamamoto, K. u. K. Hayashi: Symp. Enzyme Chem. 18, 21 (1968).

9. Aoyagi, T., A. Takeuchi, A. Matsuzaki, K. Kawamura, S. Kondo, M. Hamada, K. Maeda, H. Umezawa: J. Antibiotics 22, 283 (1969).

10. Suda, H., T. Aoyagi, M. Hamada, T. Takeuchi, H. Umezawa: J. Antibiotics 25, 263 (1972).

11. Umezawa, H., T. Aoyagi, H. Morishima, S. Kunimoto, M. Matsuzaki, M. Hamada, T. Takeuchi: J. Antibiotics 23, 425 (1970).

12. Umezawa, H., T. Aoyagi, H. Morishima, M. Matsuzaki, M. Hamada, T. Takeuchi: J. Antibiotics 23, 259 (1970).

13. Aoyagi, T., H. Umezawa: In Proteases and Biological Control, Cold Spring Harbor Conferences on Cell Proliferation, Vol. 2, 429 (1975), Cold Spring Harbor Laboratory.

14. Wingender, W., in H. Fritz, H. Tschesche, L. J. Greene u. E. Truscheit (eds.): Bayer Symposium V, „Proteinase Inhibitors“ S. 548, Berlin, Heidelberg, New York 1974, Springer Verlag.

15. Wingender, W., H. v. Hugo, W. Frommer, D. Schäfer: J. Antibiotics 28, 611 (1975).

16. Tatsuta, K., N. Mikami, K. Fujimoto, S. Umezawa, T. Aoyagi, H. Umezawa: J. Antibiotics 26, 625 (1973).

17. Fujimoto, K., K. Tatsuta, T. Tsuchiya, S. 
Umezawa, H. Umezawa: J. Antibiotics 27, 685 (1974).

18. Aoyagi, T., Y. Yagisawa, M. Kumagai, M. Hamada, H. Morishima, T. Takeuchi, H. Umezawa: J. Antibiotics 26, 539 (1973).

19. Umezawa, H., T. Miyano, T. Murakami, T. Takita, T. Aoyagi, T. Takeuchi, H. Naganawa, H. Morishima: J. Antibiotics 26, 614 (1973).

20. Kakinuma, A., T. Kanamura, H. Sugino, T. Asano, M. Yoneda: USP 3907764 v. 23. 9. 1974, Priorität Japan 9. 4. 1973.

21. Kojima, S., K. Nakamura, T. Koide, S. Ogino: DOS 2640420 v. 31. 3. 1977, Priorität Japan 12. 9. 1975.

22. Kakinuma, A., H. Sugino, N. Moriya, M. Isono: USP B 328077 b. 13. 4. 1972, Priorität Japan 8. 2. 1972.

22a. Sugino, H., A. Kakinuma, S. Iwanaga: J. Biol. Chem. 253, 1546 (1978).

23. Murao, S., S. Sato: Agrar. Biol. Chem. 36, 160 (1972).

23a. Murao, S., T. Watanabe: Agr. Biol. Chem. 41, 1313 (1977).

24. Ikenaka, T., S. Odani, M. Sakai, Y. Nabeshima, S. Sato, S. Murao: J. Biochem. 76, 1191 (1974).

25. Holzer, H., in S. Shaltiel (ed.): Metabolic Interconversion of Enzymes 1975. 4th Intern. Symp., Avad (Israel), S. 168-174, Berlin, Heidelberg, New York 1976, Springer Verlag.

26. Umezawa, H., T. Aoyagi, A. Okura, H. Morishima, T. Takeuchi, Y. Okami: J. Antibiotics 26, 787 (1973).

27. Okura, A., H. Morishima, T. Takita, T. Aoyagi, T. Takeuchi, H. Umezawa: J. Antibiotics 28, 337 (1975).

28. Suda, H., T. Aoyagi, T. Takeuchi, H. Umezawa: J. Antibiotics 26, 621 (1973).

29. Umezawa, H., T. Aoyagi, H. Suda, M. Hameda, T. Takeuchi: J. Antibiotics 29, 97 (1976).

30. Suda, H., T. Takita, T. Aoyagi, H. Umezawa: J. Antibiotics 29, 100 (1976).

31. Nakamura, T., H. Yasuda, A. Obayashi, O. Tanabe, S. Matsumura, F. Ueda, K. Ohata: J. Antibiotics 28, 477 (1975).

32. Chimura, H., T. Sawa, T. Takita, M. Matsuzaki, T. Takeuchi, T. Nagatsu, H. Umezawa: J. Antibiotics 26, 112 (1973).

33. Chimura, H., T. Sawa, Y. Kumada, F. Na- kamura, M. Matsuzaki, T. Takita, T. Takeuchi, H. Umezawa: J. Antibiotics 26, 618 (1973).

34. Kumada, Y., H. Naganawa, H. Jinuma, M. Matsuzaki, T. Takeuchi, H. Umezawa: J. Antibiotics 29, 882 (1976).

35. Chimura, H., T. Sawa, Y. Kumada, H. Naganawa, M. Matsuzaki, T. Takita, M. Hamada, T. Takeuchi, H. Umezawa: J. Antibiotics 28,619 (1975).

36. Umezawa, H., H. Tobe, N. Shibamoto, F. Nakamura, K. Nakamura, M. Matsuzaki, T. Takeuchi: J. Antibiotics 28, 947 (1975).

37. Tobe, H., H. Naganawa, T. Takita, T. Takeuchi, H. Umezawa: J. Antibiotics 29, 623 (1976).

38. Kitano, K.: J. Takeda Res. Lab. 38, 105 (1977).

39. Brown, A. G., D. Butterworth, M. Cole, G. Hanscomb, J. D. Hood, C. Reading, G. N. Rolinson: J. Antibiotics 29, 668 (1976). 40. Aoyagi, T., T. Kamamoto, K. Kojiri, F. Kojima, M. Hamada, T. Takeuchi, H. Umezawa: J. Antibiotics 31, 244 (1978).

41. Takeuchi, T., H. Chimura, M. Hamada, H. Umezawa, O. Yoshioka, N. Oguchi, Y. Takahashi, A. Matsuda: J. Antibiotics 28, 737 (1975).

42. Takeuchi, T., K. Ogawa, H. Jinuma, H. Suda, K. Ukita, T. Nagatsu, M. Kato, $\mathrm{H}$. Umezawa, O. Tanabe: J. Antibiotics 26, 162 (1973).

43. Furutani, Y., M. Shimada, M. Hamada, T. Takeuchi, H. Umezawa: J. Antibiotics 28, 558 (1975).

44. Puls, W., U. Keup, H. P. Krause, G. Thomas: Naunyn-Schmideberg's Arch. of Pharmacology Suppl. 302, 1978, R 7, Nr. 28.

45. Rao, M. N., K. S. Schurpalekav, O. E. Sudaravalla: Ind. J. Biochem. 4, 185 (1967).

46. Marshall, J. J., C. M. Lauda: J. Biol. Chem. 250, 8030 (1975).

47. Kneen, E., R. M. Sandstedt: J. Amer. Chem. Soc. 65, 1247 (1943).

48. Schmidt, D., W. Puls: DOS 2003934 v. 5. 8. 1971, Prioritätsdatum 26. 1. 1970.

49. Puls, W., U. Keup: Diabetologia 9, 97 (1973).

50. Ueda, S., Y. Koba: USP 3806421 v. 23. 4. 1974, Prior. Japan 31. 5. 1971.

51. Goto, H., T. Junkei, M. Amano: Jap. Of- 
fenlegungsschr. v. 24. 6. 1975, Priorität 20. 11. 1973.

52. Murao, S.: Jap. Offenlegungsschr. 50-123891 v. 29. 9. 1975, Priorität 15. 3. 1974.

53. Ueda, K., S. Gochyo: Jap. Offenlegungsschr. 54990 v. 14. 5. 1976, Prioritär 5. 11. 1974.

54. Belloc, A., J. Florent, J. Lunel, D. Mancy, J. C. Palla: DOS 2702417 v. 28. 7. 1977, Priorität 22. 1. 1976.

55. Koba, V., M. Najima, S. Ueda: Agr. Biol. Chem. 40, 1167 (1976).

56. Inouye, S., T. Tsuoka, T. Ito, T. Niida: Tetrahedron 23, 2125 (1968).

57. Niwa, T., S. Inouye, T. Tsuruoka, Y. Koaze, T. Niida: Agr. Biol. Chem. 34, 966 (1970).

58. Reese, E. T., F. W. Parrish, M. Ettlinger: Carbohydrate Research 18, 381 (1971).

59. Aoyagi, T., M. Kumagai, T. Hazato, M. Hamada, T. Takeuchi, H. Umezawa: J. Antibiotics 28, 555 (1975).

60. Kumagai, M., H. Naganawa, T. Aoyagi, H. Umezawa, H. Nakamura, Y. Jitaka: J. Antibiotics 28, 876 (1975).

61. Aoyagi, T., T. Hazato, M. Kumagai, M. Hamada, T. Takeuchi, H. Umezawa: J. Antibiotics 28, 1006 (1975).

62. Umezawa, H., T. Aoyagi, T. Komiyama, H. Morishima, M. Hamada, T. Takeuchi: J. Antibiotics 27, 963 (1974).

63. Frommer, W., W. Puls, D. Schmidt: DOS 2209833 v. 6. 9. 1973, Priorität: 1. 3. 1972.

64. Frommer, W., W. Puls, D. Schmidt: DOS 2209834 v. 6. 9. 1973, Pirorität: 1. 3. 1972.

65. Ruyssen, R., in R. Ruyssen (ed.): Symposium on Pharmaceutical Enzymes and their Assay (University of Ghent, May 24., 1968), S. 99, Universitaire Pers. Ghent, Belgium 1969.

66. Frommer, W., B. Junge, U. Keup, L. Müller, W. Puls, D. Schmidt: DOS 2347782 v. 10. 4. 1975, Priorität: 22. 9. 1973.

67. Frommer, W., W. Puls, D. Schmidt: DOS 2209832 v. 6. 9. 1973, Priorität: 1. 3. 1972.
68. Schmidt, D. D., W. Frommer, B. Junge, L. Müller, W. Wingender, E. Truscheit, D. Schäfer: Naturwissenschaften 64,535 (1977).

69. Horii, S., T. Iwasa, E. Mizuta, Y. Kameda: J. Antibiotics 24, 59 (1971).

70. Stevens, C. L. et al.: J. Org. Chem. 31, 2822 (1966).

71. Puls, W., W. Frommer, H. Hulpke, B. Junge, U. Keup, J. Kurz, L. Müller, D. Schäfer, D. Schmidt, D. Wendisch, W. Wingender, C. Wünsche: Vortrag gehalten von Puls, W. auf dem Diabetologen Kongress 1974 in Travemünde.

72. Puls, W., U. Keup: Recent Advances in Obesity Research: I Proceedings of the 1st International Congress on Obesity, October 8-11, London 1974, Newman Publishing Ltd.

73. Puls, W., U. Keup, H. P. Krause, G. Thomas, F. Hoffmeister: Naturwissenschaften 64, 536 (1977).

74. Oeding, V., W. Pfaff, L. Vertesy, H. L. Weidemüller: DOS 2701890 v. 27. 7. 1978, Priorität: 19. 1. 1977.

74a. Oeding, V., H. Neubauer, L. Vertesy, H. L. Weidenmüller: DOS 2716050 v. 19. 10. 1978. Priorität 9. 4. 1977.

75. Frommer, W., L. Müller, D. Schmidt, W. Puls, H.-P. Krause, U. Heber: DOS 2658563 v. 29. 6. 1978, Priorität: 23. 12. 1976.

76. Omora, S., H. Ohno, T. Saheki, M. Yoshida, A. Nakagawa: Biochem. Biophys. Res. Com. 83, 704 (1978).

77. Kondo, S., K. Uotani, M. Myamoto, T. Hazato, H. Naganawa, T. Aoyagi, H. Umezawa: J. Antibiotics 31, 797 (1978).

Adresse: Prof. Dr. W. Frommer, Bayer A.G., Pharma Forschungszentrum, Mikrobiologische Abteilung, Postfach 101708, D-5600 Wuppertal 1 